\title{
The effects of the natural antioxidant quercetin and anions of the Hofmeister series on liposomes marked with chlorophyll $a$
}

\author{
Magdalena Drăguşin ${ }^{1}$, Laura Țugulea ${ }^{2}$ and Constanța Ganea ${ }^{1}$ \\ ${ }^{1}$ Department of Biophysics, University of Medicine and Pharmacy Carol Davila, 8 Eroii Sanitari Blvd., 050474 Bucharest, \\ Romania; E-mail: magdadragusin@yahoo.com \\ ${ }^{2}$ University of Bucharest, Faculty of Physics, 405 Atomiștilor Str., 077125 Măgurele, Romania
}

\begin{abstract}
The unilamellar liposomes of dimyristoylphosphatidylcholine (DMPC), marked with chlorophyll $a$ (Chla), have been chosen as suitable models in studies aimed at determining the effects of natural antioxidant quercetin (QCT) and Hofmeister series anions on lipid bilayers. The variations of steady state fluorescence emission intensity of Chla have been recorded and the values of Chla fluorescence anisotropy, under constant temperature and viscosity, at $\mathrm{pH}=7.3-7.4$ have been measured. Two types of experiments have been performed. In the first type of experiment, the concentration of anions was maintained constant and the concentration of QCT was varied (from 0 to $100 \mu \mathrm{mol} / \mathrm{l}$ ). In the second type of experiment, the concentration of QCT was constant $(30 \mu \mathrm{mol} / \mathrm{l})$ and the concentration of anions was varied (from 0 to $152 \mathrm{mmol} / \mathrm{l}$ ). The quenching of Chla fluorescence by QCT pleads in favor of QCT insertion at interface water-lipid, in the vicinity of the polar heads of lipids from liposomal bilayer at physiological $\mathrm{pH}$ and temperature. Fluorescence anisotropy of Chla in liposomes brought more evidences for QCT localizations at lipid/water interface. Chla is sensing a more rigid microenvironment when QCT is added to the lipid bilayer and specific effects of the Hofmeister series anions.
\end{abstract}

Key words: Quercetin - Hofmeister anions - DMPC liposomes - Chlorophyll $a$ - Spectrophotofluorimetry

\begin{abstract}
Abbreviations: Chla, chlorophyll $a$; DMPC, dimyristoylphosphatidylcholine; DMSO, dimethyl sulfoxide; $\mathrm{I}_{\mathrm{max}}$, the relative intensity emission maximum; PBS, phosphate buffer solution; ROS, reactive oxygen species; SUVs, small unilamellar vesicles; QCT, quercetin.
\end{abstract}

\section{Introduction}

Multiple biological events that occur at the cellular membrane and interactions of membrane components with the cytoskeleton could be severely affected when damages induced by reactive oxygen species (ROS) occur (Oteiza et al. 2005). It is known that the increased release of free radicals and ROS affects the membrane fluidity, the cellular $\mathrm{Ca}^{2+}$ homeostasis and induces lipid peroxidation leading finally to the cell death (Hollán et al. 1996).

Flavonoids like quercetin (QCT) provide antioxidant properties, so that, at the level of cell membrane, they have

Correspondence to: Magdalena Drăguşin, Department of Biophysics, University of Medicine and Pharmacy Carol Davila, 8 Eroii Sanitari Blvd., 050474, Bucharest, Romania

E-mail:magdadragusin@yahoo.com the capacity to prevent the lipid peroxidation induced by free radicals. QCT (3,3,4'5,7-pentahydroxyflavone) is a natural flavonol, found in many ingredients of the human diet, consumed at highest levels due to its presence in tea, onions, apples (Hertog et al. 1996; Pawlikowska-Pawlega et al. 2007).

The antioxidant properties are determined by chemical structure of QCT, in particular by the presence and location of the hydroxyl $(-\mathrm{OH})$ substitutions and the catechol-type B-ring (Wang et al. 2006). Various authors indicated that QCT, which is characterized by a hydroxylation pattern of 3, 5, 7, 3', and 4' and a catechol B-ring, possesses all the structural elements characteristic of an antioxidant and by exerting its antioxidant activity, QCT may be converted into reactive products (Metodiewa et al. 1999; Rietjens et al. 2005; Harwood et al. 2007).

Other important factors that contribute to the antioxidant capacity of QCT are the orientation in the membrane lipid bilayer, the degree of incorporation, the uniformity of dis- 
tribution (Saija et al. 1995; Arora et al. 2000). The $\mathrm{pH}$ of the media influences the QCT location within the membrane and its resulting restrictions on the fluidity of membrane components may sterically block the diffusion of free radicals, thus decreasing the kinetics of free radical reactions (Arora et al. 2000; Barros et al. 2001; Ilie et al. 2009 a,b). The embedding of QCT in biological and in model membranes depends, also, on the $\mathrm{pH}$ of the media. At acidic $\mathrm{pH}, \mathrm{QCT}$ is deeply embedded in planar lipid bilayers, while at physiological $\mathrm{pH}$ it interacts with the polar head groups at the water-lipid interface (Movileanu et al. 2000; Pawlikowska-Pawlega et al. 2003).

The incorporation is affected by electrostatic interactions, the formation of hydrogen bonds with the polar groups of phospholipids, hydrophobic interactions with fatty acyl chains, and by the molecular geometry of phospholipids (Rego et al. 1995). More general, it is known that the antioxidant efficiency of all flavonoids increases in direct order to their flexibility to conformational changes and in an inverse order to their ability to establish hydrogen bonds. The hydrophobicity of flavonoids and hence their ability to interact with biological membranes is one of the main determinants of their pharmacological activity. So, it was experimentally demonstrated that an inverse correlation between the number of hydroxyl groups and flavonoid hydrophobicity does exist (Rego et al. 1995; Arora et al. 2000; van Dijk et al. 2000; Scheidt et al. 2004). This rule is also applicable for prediction of flavonoids localization in the lipid bilayer as it was demonstrated by NMR spectroscopy (Scheidt et al. 2004). The location of flavonoid molecules in the hydrophobic region or in the polar membrane interface depends on hydrogen bonding of the hydroxyl groups. Molecules of QCT could be comparable with cholesterol, regarding the localization and the influence on the bilayer, increasing the viscosity of lipid bilayer (Tsuchiya et al. 2002; Tarahovsky et al. 2008).

Lyotropic ions or anions of the Hofmeister series induce changes of lipid phase behavior or surface potential and dipolar moment alterations in lipid membranes and liposomes (Sanderson et al. 1991; Clarke et al. 1997, 1999; Koynova et al. 1997). The Hofmeister series are usually given in terms of the ability of the ions to stabilize the structure of proteins, which is generally more pronounced for anions than for cations. The typical ordering of the anion series is: $\mathrm{SO}_{4}{ }^{2-}, \mathrm{HPO}_{4}{ }^{2-}, \mathrm{OH}^{-}$, $\mathrm{F}^{-}, \mathrm{HCOO}^{-}, \mathrm{CH}_{3} \mathrm{COO}^{-}, \mathrm{Cl}^{-}, \mathrm{Br}^{-}, \mathrm{NO}_{3}{ }^{-}, \mathrm{I}^{-}, \mathrm{SCN}^{-}, \mathrm{ClO}_{4}^{-}$. The ions to the left of $\mathrm{Cl}^{-}$are referred to as kosmotropes, while those to its right are called chaotropes. These terms originally referred to an ion's ability to alter the hydrogen bonding network of water. The kosmotropes, which were believed to be 'water structure makers', are strongly hydrated and have stabilizing and salting-out effects on proteins and macromolecules. On the other hand, chaotropes ('water structure breakers') are known to destabilize folded proteins and give rise to salting-in behavior (Collins et al. 1985; Ru et al. 2000).

In the close neighborhood of lipid membranes, the chaotropic ions show the tendency to insert themselves into the hydrophobic membrane (salting-in effect), while the kosmotropic ions distribute in the surrounding water (salting-out effect) (Clarke et al. 1999; Zhang et al. 2006; Garcia-Celma et al. 2007).

Although Hofmeister effects for macromolecules in aqueous solution are ubiquitous, the molecular-level mechanisms by which ions operate has not yet been clarified and no generally accepted explanation exists at the molecular level.

Nowadays, anions of the Hofmeister series are found in many pharmaceutical and food products, resulting from the preparation and conservation methods, as well as from chemical fertilizers based on nitrates. Due to this fact, the way in which QCT and anions may affect cellular membranes and their compounds is of interest, and has implications on their function.

The effects of QCT on the lipid membrane in the presence of Hofmeister anions and the manner in which the QCT could affect the properties of lipid membranes, induced by some of these anions: sulphate, chloride, nitrate and perchlorate, have been investigated by using liposomes as biomembrane models. The liposomes with chlorophyll $a$ (Chla) proved to be useful cell membrane models for investigating the antioxidant capacity of QCT in the presence of Hofmeister anions. Due to the spectral features (strong visible absorption and fluorescence), Chla incorporated into the liposomal lipid bilayer was used as a molecular spectral sensor to monitor the effects of the Hofmeister series anions in the presence of natural flavonoid QCT on liposomes.

\section{Materials and Methods}

\section{Materials and reagents}

QCT (Sigma) has been prepared as $10 \mathrm{mmol} / \mathrm{l}$ and $20 \mathrm{mmol} / \mathrm{l}$ stock solution in dimethyl sulfoxide (DMSO).

Sodium chloride $(\mathrm{NaCl})$, sodium nitrate $\left(\mathrm{NaNO}_{3}\right)$, sodium perchlorate $\left(\mathrm{NaClO}_{4}\right)$ (Merck) and sodium sulphate $\left(\mathrm{Na}_{2} \mathrm{SO}_{4}\right.$; Sigma) salts were prepared as stock solutions of concentration $4 \mathrm{~mol} / \mathrm{l}$ in phosphate buffer solution (PBS) $0.066 \mathrm{~mol} / \mathrm{l}, \mathrm{pH}=7.3-7.4$.

Dimyristoylphosphatidylcholine (DMPC; $\mathrm{C}_{36} \mathrm{H}_{72} \mathrm{NO}_{8} \mathrm{P}$; $\left.\mathrm{MW}=677.940 \mathrm{~g} / \mathrm{mol} ; \mathrm{T}_{\mathrm{c}}=23^{\circ}-24^{\circ} \mathrm{C}\right)$, the lipid used for preparation of all liposomes populations, was purchased from Sigma Aldrich (Germany).

All solutions were prepared using analytical grade reagents and purified water with conductivity $\leq 0.1 \mu \mathrm{S} \cdot \mathrm{cm}^{-1}$ from a Millipore Milli-Q system (Billerica, Massachusetts, USA).

\section{Liposomes preparation}

Liposomes were prepared according to the thin-film hydration method (R.R.C. New 1990), with little modifications. The lipid DMPC was dissolved in chloroform together with Chla 
$($ Chla/lipid molar ratio $=1 / 100)$. Chla was extracted from fresh spinach leaves by a chromatographic method using a powdered sugar column and organic solvents (petroleum ether, n-propanol and ethanol) as mobile phase, according to the procedure of Strain and Svec (1966). The purity and concentration of Chla were monitored by using VIS absorption and emission spectra of Chla in diethyl ether and spectral criteria (Strain and Svec 1966). Chloroform solvent, from solutions containing lipid and Chla in specified concentration, was removed in a rotary evaporator Heildolph 94200, 60-90 rpm (Bioblock Scientific, Illkirch Cedex, France), using a vacuum pump. The lipid films were hydrated, during 1 hour, in PBS ( $\mathrm{pH}=7.3-7.4)$. The resulted suspensions of multilamellar vesicles were mechanically mixed with Vibrax stirrer, $200 \mathrm{rpm}$ (Milian, USA) and then sonicated, $15 \mathrm{~min}$, using a titanium probe sonicator Hielscher, UP $100 \mathrm{H}$ (Hielscher Ultrasonics $\mathrm{GmbH}$, Teltow, Germany) to produce small unilamellar vesicles (SUVs) as stated in the literature (R.R.C. New 1990). Spectral criteria in UV-VIS range of absorption developed for Chla liposomes were used to rapid check the dimension and unilamellarity of liposomes (Bărbîntă-Pătrascu et al. 2008). All the operations have been performed at $36.7^{\circ} \mathrm{C}$, therefore above the critical temperature $\left(\mathrm{T}_{\mathrm{c}}\right)$ of the lipid (lipids being in the liquid crystal phase, the probability to obtain liposomes without defects is higher). The suspensions were centrifuged $45 \mathrm{~min}, 20000 \times g$, with SIGMA 2-16 K centrifuge (SciQuip Ltd Merrington, Shrewsbury Shropshire, UK) in order to remove the titanium traces and to obtain homogeneous vesicle populations. Only the supernatants were used in investigations.

\section{Spectral measurements}

The optical absorption spectra were obtained on a double beam UV-VIS spectrophotometer Lambda 2S Perkin Elmer using PECSS software. Fluorescence experiments in steady state were done and fluorescence polarization measurements were also performed. Perkin Elmer LS 55 fluorescence spectrometer was used (Waltham, Massachusetts, USA), fitted with a biokinetic accessory providing continuous monitoring of the temperature in the cuvette and stirring of liposome suspension. The LS 55 fluorescence spectrometer was equipped with R928 photomultiplier, red-sensitive photomultiplier fitted for collection of emission data above $630 \mathrm{~nm}$. The excitation spectra are automatically corrected. Slits of $5 \mathrm{~nm}$ were used both for excitation and emission. Wavelength accuracy of the instrument is $\pm 1.0 \mathrm{~nm}$ and wavelength reproducibility is $\pm 0.5 \mathrm{~nm}$. An ultra thermostat/circulator Julabo ED 5 (Julabo Labortechnik GmbH 77960 Seelbach/Germany) with accuracy of $\pm 0.3 \mathrm{~K}$ ensured the constant temperature of $36.7^{\circ} \mathrm{C}$ (the physiological temperature of human body) in the cuvette, during all fluorescence measurements.
Procession of all spectral data has been done using the software Origin 8.0.

\section{Methodology}

The Hofmeister salts, used in two types of experiments, presented a common cation $\left(\mathrm{Na}^{+}\right)$and the following anions: kosmotrop $\mathrm{SO}_{4}{ }^{2-}$, neutral $\mathrm{Cl}^{-}$and chaotrops $\mathrm{NO}_{3}{ }^{-}, \mathrm{ClO}_{4}{ }^{-}$. Suspensions of freshly prepared unilamellar liposomes marked with Chla have been used in both types of experiments. QCT and anions have been added to SUVs suspension leading to the desired concentration. Three independent repetitions for each type of experiment have been done and the records were used to calculate the average. The further processing of the data was based on the average values (Figs. 2-6 and Table 1).

In the first type experiment, the concentration of anions was maintained constant ( 16 and $52 \mathrm{mmol} / \mathrm{l}$, respectively) and the concentration of QCT was varied (from 0 to 100 $\mu \mathrm{mol} / \mathrm{l})$. The control in type I experiments was represented by Chla liposomes in the presence of QCT (from 0 to 100 $\mu \mathrm{mol} / \mathrm{l}$ ) and without anions. Aliquots of QCT stock DMSO solution have been added in the cuvette solution/suspension to obtain a concentration range of $0-100 \mu \mathrm{mol} / \mathrm{l}$ for every spectral measurement. The DMSO concentration $(\mathrm{v} / \mathrm{v})$ in the cuvette did not exceed $0.5 \%$.

In the second type experiment, the concentration of QCT was constant $(30 \mu \mathrm{mol} / \mathrm{l})$ and the concentration of anions was varied (from 0 to $152 \mathrm{mmol} / \mathrm{l}$ ). The control in type II experiments was represented by Chla liposomes in the presence of anions (from 0 to $152 \mathrm{mmol} / \mathrm{l}$ ) and without QCT. Aliquots of anions stock solutions have been added in the cuvette suspension/solution to achieve the desired concentrations.

\section{Results}

\section{Steady state fluorescence experiments}

Chla fluorescence emission in liposomes suspension was measured in the presence of QCT and anions. The maximum at $430 \mathrm{~nm}$ in the Soret band was used as excitation wavelength for all emission spectra. Chla was used as a fluorescent probe and molecular sensor in all the fluorescence experiments. Always control spectra have been recorded, using liposomes with Chla.

As mentioned previously, two types of experiments have been done, by varying either the concentration of QCT added to liposome suspensions, in the presence of anions (16 or $52 \mathrm{mmol} / \mathrm{l})$ or anion concentration, in the presence of fixed QCT concentration $(30 \mu \mathrm{mol} / \mathrm{l})$. In both types of experiments, no wavelength shifts in the 


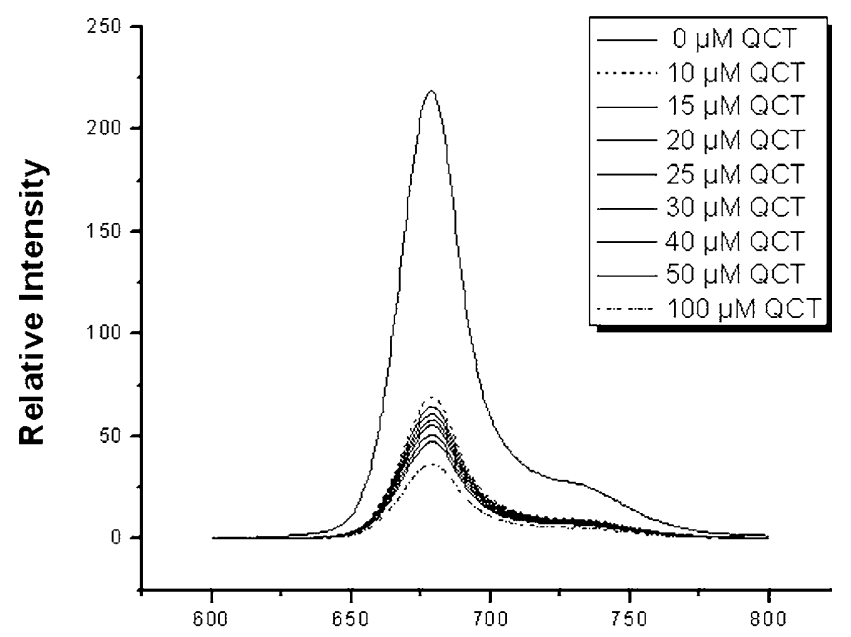

Figure 1. Fluorescence emission spectra of Chla in liposomes in the presence of different concentrations of QCT $\left(\lambda_{\text {exc }}=430 \mathrm{~nm}\right)$.

emission spectra have been detected within instrumental resolution limit.

\section{Type I experiments}

The relative intensity emission maximum $\left(\mathrm{I}_{\max }\right)$ at 678 $\mathrm{nm}$ was monitored and in Fig. 1 the influence of QCT is presented. It is known that Chla is located in the liposome lipid bilayer with the macrocycle at the interface with the water phase, in the vicinity of the lipid polar heads and the phytol in the hydrophobic lipid chains region (Țugulea et

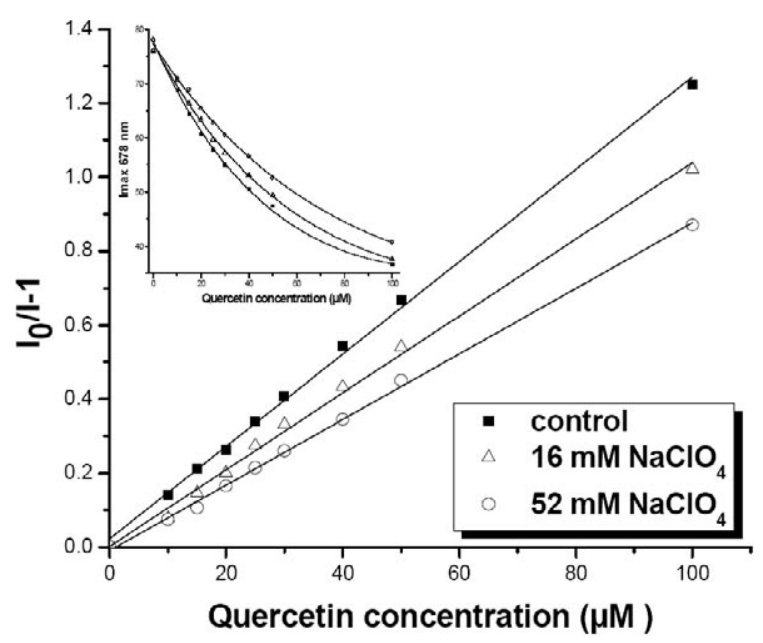

Figure 2. Stern-Volmer plot for liposomes with Chla, in the presence of $\mathrm{NaClO}_{4}$ and different concentrations of QCT. Insertion: plot of the fluorescence emission intensity of Chla in liposomes in the presence of $\mathrm{NaClO}_{4}$ and different concentrations of QCT $\left(\lambda_{\text {exc }}=430 \mathrm{~nm}, \lambda_{\mathrm{em}}=678 \mathrm{~nm}\right)$. al. 1996). The relative intensity of Chla is decreasing in the presence of QCT, thus QCT is acting as a quencher for Chla fluorescence in liposomes.

The same type of behavior of the relative emission intensity was observed when QCT was added in the presence of all the anions: $\mathrm{SO}_{4}{ }^{2-}, \mathrm{Cl}^{-}, \mathrm{NO}_{3}{ }^{-}$and $\mathrm{ClO}_{4}^{-}$.

Thus, QCT acts like a quencher of the fluorescence of Chla, without and in the presence of all anions species, and the quenching respects the Stern-Volmer equation (Lakowicz 1983):

$\Phi_{0} / \Phi=\mathrm{I}_{0} / \mathrm{I}=1+\mathrm{K}_{\mathrm{q}} \tau_{0}[\mathrm{Q}]=1+\mathrm{K}_{\mathrm{SV}}[\mathrm{Q}]$

where: $\mathrm{I}_{0}$ and $\mathrm{I}$ are the steady-state fluorescence intensities in the absence and presence of quencher, respectively; $\mathrm{K}_{\mathrm{SV}}=$ $\mathrm{K}_{\mathrm{q}} \tau_{0}$ is the Stern-Volmer constant; $\tau_{0}$ is the fluorescence lifetime in the absence of the Q; [Q] is the quencher concentration; $\mathrm{K}_{\mathrm{q}}$ is the bimolecular quenching constant.

Fig. 2 presents the Stern-Volmer plot for liposomes with Chla, when QCT was added in the presence of the anion $\left(\mathrm{ClO}_{4}{ }^{-}\right)$, the control being represented by liposomes with Chla, without anion. The data in the insertion of Fig. 2 are fitted well with exponential curve.

A linear Stern-Volmer plot is generally indicative of a single class of fluorophores, all equally accessible to quencher. If two fluorophore populations are present, and one is not accessible to the quencher, then Stern Volmer plots deviate from linearity toward $x$ axis. Much more, a linear SternVolmer plot results either in the case of dynamic or static quenching (Lakowicz 1983).

\section{Type II experiments}

Fig. 3 presents the variation of relative intensity of Chla fluorescence in type II experiments: in the presence of $30 \mu \mathrm{mol} / 1$ QCT and varied anion concentrations. Some common features for all anions, excluding $\mathrm{ClO}_{4}{ }^{-}$, are notable: Chla fluorescence presents a slight decrease in the presence of QCT. The variation of the relative intensity of Chla in the presence of $30 \mu \mathrm{mol} / \mathrm{l} \mathrm{QCT}$ and the three anions: $\mathrm{SO}_{4}{ }^{2-}$, $\mathrm{Cl}^{-}, \mathrm{NO}_{3}{ }^{-}$, is fitted by a decreasing exponential curve. In the absence of QCT (control experiment), the presence of varied concentrations of anions has a very small influence on Chla fluorescence.

\section{Fluorescence anisotropy}

All fluorescence anisotropy experiments have been done at temperature of $36.7^{\circ} \mathrm{C}$, therefore above the transition temperature of DMPC (phospholipid used in all liposome preparations). The emission anisotropy values for Chla in liposomes were recorded using the following wavelengths: $\lambda_{\mathrm{em}}=678 \mathrm{~nm}$ and $\lambda_{\mathrm{exc}}=430 \mathrm{~nm}$. For each measurement the correction for $\mathrm{G}$ factor (the ratio of the sensitivities of the detection system for vertically and horizontally polar- 
A

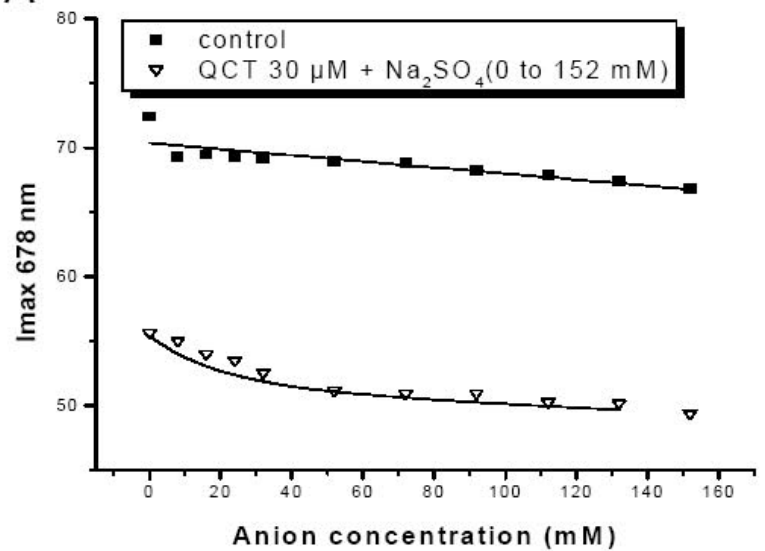

C



B

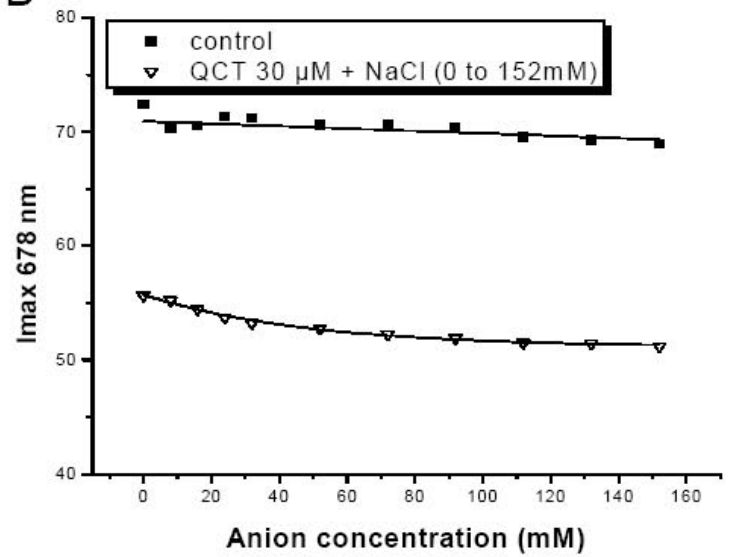

$\mathrm{D}$

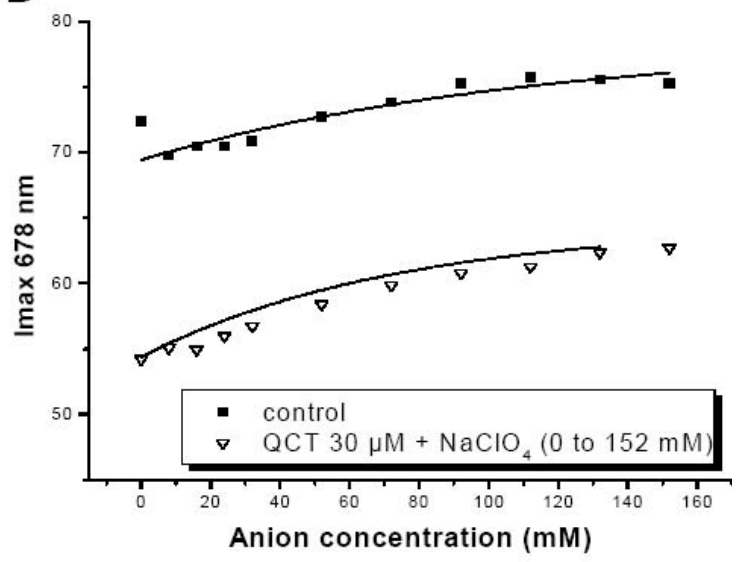

Figure 3. Fluorescence emission intensity of Chla in liposomes in the presence of QCT ( $30 \mu \mathrm{mol} / \mathrm{l})$ and different concentrations of anions: $\mathrm{Na}_{2} \mathrm{SO}_{4}(\mathrm{~A}), \mathrm{NaCl}(\mathrm{B}), \mathrm{NaNO}_{3}(\mathrm{C}), \mathrm{NaClO}_{4}(\mathrm{D})\left(\lambda_{\mathrm{exc}}=430 \mathrm{~nm}, \lambda_{\mathrm{em}}=678 \mathrm{~nm}\right)$.

ized light) was applied. Control experiments for monitoring the possible dilution effect have been done. The addition of buffer solution in volumes corresponding to those of anions aliquots resulted in non significant effects on Chla emission anisotropy and therefore no dilution corrections were necessary.

Fig. 4 presents the anisotropy plot of liposomes with Chla in the presence of the most chaotropic anion, $\mathrm{ClO}_{4}{ }^{-}$and varying concentrations of QCT (type I experiment). At the same concentration of QCT, the presence of all anions resulted in lower values for the anisotropy emission. $\mathrm{ClO}_{4}{ }^{-}$was chosen because his effect was more evident in this respect.

The fluorescence anisotropy plots in type II experiments are presented in Fig. 5. The presence of anions and their concentrations does not affect at all the anisotropy emission value of Chla, which is rather low. The presence of $30 \mu \mathrm{mol} / \mathrm{l}$ of QCT leads to increasing values for emission anisotropy, no matter the anion nature.



Figure 4. Anisotropy plot of liposomes with Chla in the presence of $\mathrm{NaClO}_{4}$ and different concentrations of QCT. 

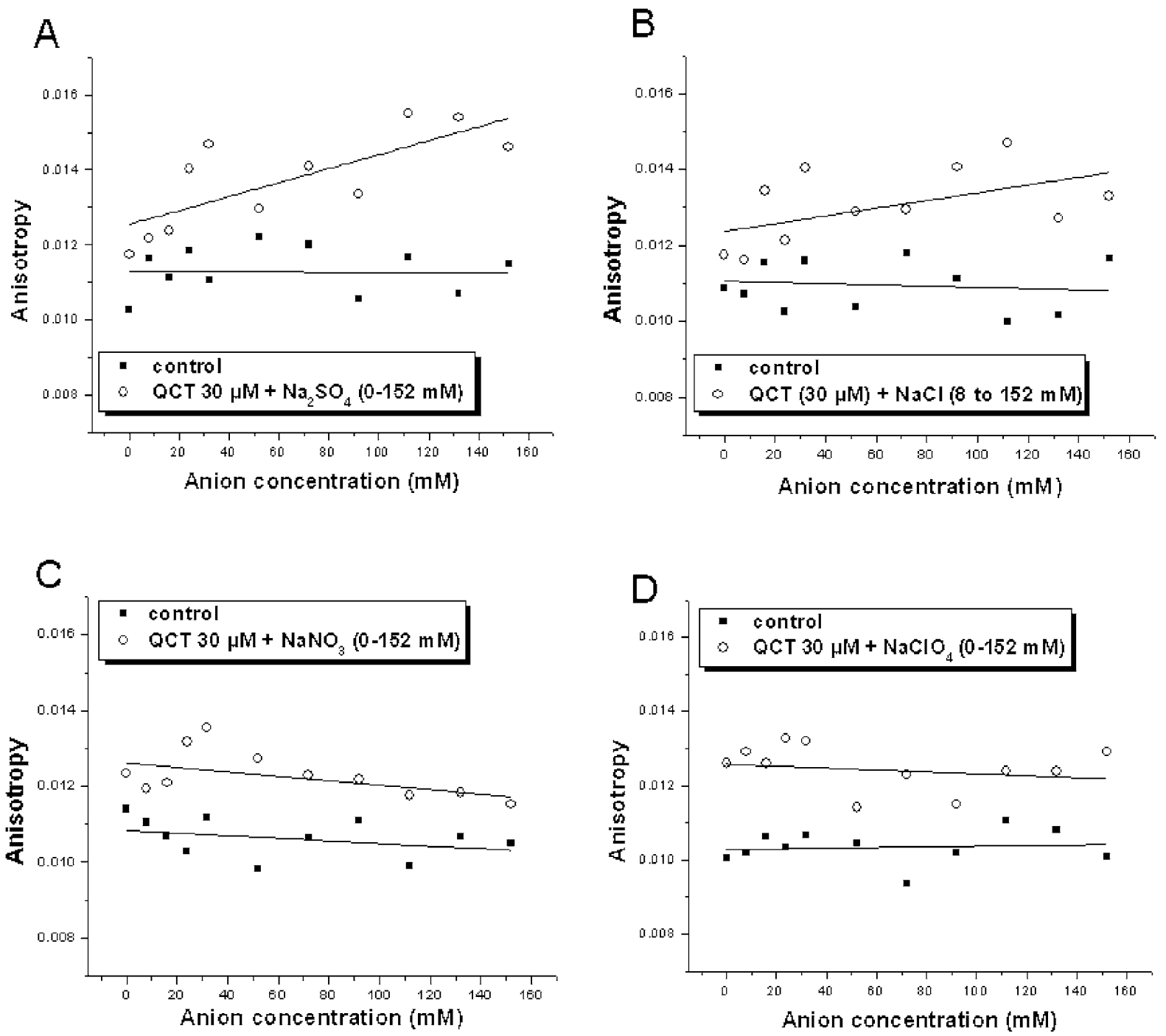

Figure 5. Anisotropy plot of liposomes with Chla in the presence of QCT $(30 \mu \mathrm{mol} / \mathrm{l})$ and different concentrations of anions: $\mathrm{Na}_{2} \mathrm{SO}_{4}$ (A), $\mathrm{NaCl}(\mathrm{B}), \mathrm{NaNO}_{3}(\mathrm{C}), \mathrm{NaClO}_{4}$ (D).

Table 1. Bimolecular quenching constants for liposomes with Chla

\begin{tabular}{|l|c|c|c|}
\hline Anion & $\begin{array}{c}\mathrm{c}_{\mathrm{A}} \\
(\mathrm{mmol} / \mathrm{l})\end{array}$ & $\mathrm{K}_{\mathrm{SV}}$ & $\begin{array}{c}\mathrm{K}_{\mathrm{q}} \\
\left(\times 10^{12} \mathrm{M}^{-1} \cdot \mathrm{s}^{-1}\right)\end{array}$ \\
\hline control & 0 & $1.125 \pm 0.000441$ & 2.25 \\
\hline \multirow{2}{*}{$\mathrm{Na}_{2} \mathrm{SO}_{4}$} & 16 & $1.076 \pm 0.000337$ & 2.16 \\
\cline { 2 - 4 } & 52 & $1.096 \pm 0.00043$ & 2.19 \\
\hline \multirow{2}{*}{$\mathrm{NaCl}$} & 16 & $1.066 \pm 0.000335$ & 2.13 \\
\cline { 2 - 4 } & 52 & $0.97 \pm 0.000279$ & 1.94 \\
\hline \multirow{2}{*}{$\mathrm{NaNO}_{3}$} & 16 & $1.011 \pm 0.000211$ & 2.02 \\
\cline { 2 - 4 } & 52 & $0.942 \pm 0.00036$ & 1.88 \\
\hline \multirow{2}{*}{$\mathrm{NaClO}_{4}$} & 16 & $1.039 \pm 0.000227$ & 2.07 \\
\cline { 2 - 4 } & 52 & $0.886 \pm 0.000125$ & 1.77 \\
\hline
\end{tabular}

$\mathrm{c}_{\mathrm{A}}$, concentration of anion; control, Chla liposomes + QCT (0-100 $\mu \mathrm{mol} / \mathrm{l})$.

\section{Discussion}

In all steady-state fluorescence experiments, QCT acts as a quencher for Chla fluorescence.

In type I experiments, a quenching obeying the Stern-Volmer equation was obtained in the presence of all anions. The Stern-Volmer plot is linear, indicating that Chla is accessible to the quencher QCT. From the slope of Stern-Volmer plot, $\mathrm{K}_{\mathrm{SV}}$ was calculated for all samples. Considering a value of 5 ns (Ilík et al. 2003) for the fluorescence lifetime of Chla in the absence of the quencher QCT, the values of bimolecular quenching constants have been calculated. The obtained values for $\mathrm{K}_{\mathrm{SV}}$ and $\mathrm{K}_{\mathrm{q}}$ are presented in Tab. 1 .

All $\mathrm{K}_{\mathrm{q}}$ values are within the range of $\sim 10^{12} \mathrm{M}^{-1} \cdot \mathrm{s}^{-1}$ indicating that the quenching process cannot be considered as one 
limited by diffusion. A dynamic quenching (collisional process) is characterized by bimolecular quenching constants in the range of $\sim 10^{10} \mathrm{M}^{-1} \cdot \mathrm{s}^{-1}$. Therefore, a static process could be considered as responsible for Chla quenching by QCT and in some cases a complex formation in the ground state could be responsible for the quenching process.

In some cases, evidence for the formation of a complex can be obtained (e.g. changes in the absorption spectrum upon complexation), but in the absence of such evidence, the interaction is likely to be non-specific and the model of an effective sphere of quenching is more appropriate (Valeur 2001). Neither changes of the absorption spectra or emission spectra (except variation of the relative intensity) have been observed on liposomes with Chla after adding QCT. An effective sphere of quenching of Chla fluorescence could be assumed and the effect of the Hofmeister series anions could be explained as regarding this sphere.

The calculated $\mathrm{K}_{\mathrm{q}}$ are presented in the Fig. 6 , as a plot. A line, representing the regression fit in the case of $\mathrm{Cl}^{-}$anion was represented in order to show the distribution of values as regarding the influence of chaotropic and kosmotropic anions on the process of Chla quenching by QCT.

$\mathrm{SO}_{4}{ }^{2-}$, kosmotrop anion, known as having a "salting-out effect" and distributed in the surrounding water, presents the highest value for $\mathrm{K}_{\mathrm{q}}$, very close to the $\mathrm{K}_{\mathrm{q}}$ value in the absence of anions. The lowest value for $\mathrm{K}_{\mathrm{q}}$ was obtained in the presence of $\mathrm{ClO}_{4}{ }^{-}$, anion belonging to the chaotrop category. These anions show the tendency to insert themselves into the hydrophobic part of the membrane ("salting-in effect").

As already proved by other investigations, at neutral pH (Movileanu et al. 2000; Pawlikowska-Pawlega et al. 2003), QCT is inserted in the lipid bilayer. The flavonoid molecule localizes in the hydrophobic region or in the polar region of the membrane interface depending on hydrogen bonding of the hydroxyl groups (Tarahovsky et al. 2008). This incorporation is affected by electrostatic interactions, the formation of hydrogen bonds with the polar groups of phospholipids, hydrophobic interactions with fatty acyl chains, and by the molecular geometry of phospholipids (Arora et al. 2000).

A good explanation for the $\mathrm{K}_{\mathrm{q}}$ values ordering in respect with Hofmeister series anions $\left(\mathrm{SO}_{4}{ }^{2-}>\mathrm{Cl}^{-}>\mathrm{NO}_{3}{ }^{-}>\mathrm{ClO}_{4}{ }^{-}\right)$ could be done considering also the positioning of Chla as regarding the lipid bilayer and QCT inserting into it. The fluorescence properties of Chla are mostly related to its porphyrin macrocycle which is positioned at the interface with surrounding water and in the vicinity of the lipid polar heads (TTugulea et al. 1996). Assuming that the sphere of quenching of Chla is surrounding mainly the Chla macrocycle, the positioning of the "quenching sphere" should be at the interface lipid bilayer-water. The anions could influence the incorporation of QCT by different type of interactions and the different values for $\mathrm{K}_{\mathrm{q}}$ could be explained in correla-

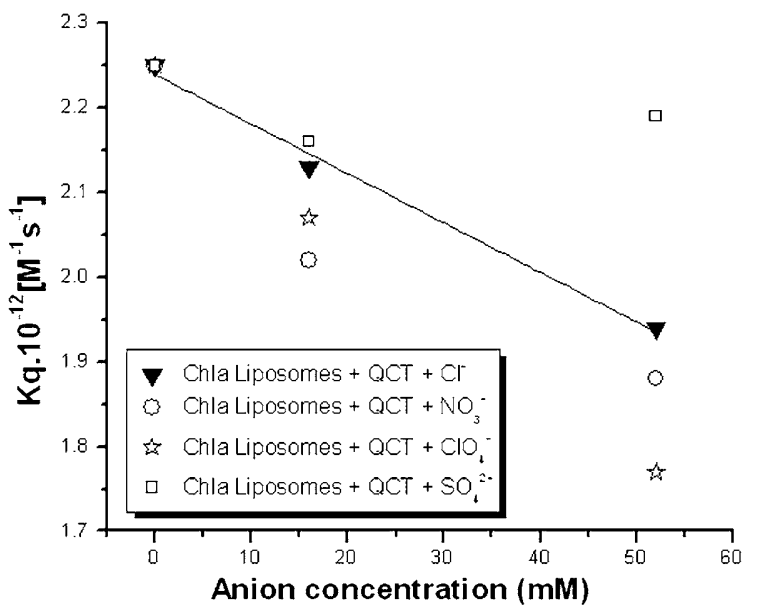

Figure 6. Dependence of the Kq value upon the anion concentration.

tion with the location of the anions. The kosmotropic anion $\mathrm{SO}_{4}{ }^{2-}$, distributed in the surrounding water, will have an influence on QCT incorporation mainly by direct electrostatic interaction, and therefore on the effective "quenching sphere". The chaotropic anions, having the tendency to enter in the hydrophobic core of the membrane, will influence the incorporation of QCT, probably by affecting the molecular geometry of phospholipids, resulting in an indirect influence of the positioning of the "quenching sphere".

In type II experiments, $\mathrm{ClO}_{4}^{-}$, the most effective among investigated chaotropic anions, induced peculiar effects on Chla fluorescence. In the control experiment (liposomes with Chla) and in the presence of QCT, the increase of anion concentration resulted in a slight increase of the relative intensity of Chla fluorescence. The slight increase of Chla fluorescence induced by higher concentrations of chaotrop ion $\mathrm{ClO}_{4}{ }^{-}$, in the presence or absence of QCT, could be explained again by the effect of this anion on the molecular geometry of phospholipids. It was shown (Clarke et al. 1999) that in the presence of this anion, having the tendency to enter in the hydrophobic core of the membrane, the increase of dipole moment of lipid is the highest. Chla fluorescence is 'sensing' the lipid molecular geometry change induced by the alteration of the dipole moment due to increasing concentrations of $\mathrm{ClO}_{4}{ }^{-}$anion. The behavior is similar when QCT is present (inserted), the only difference being lower values of the emission relative intensity due to QCT quenching effect.

The fluorescence anisotropy results are supporting the fluorescence emission results.

Chla incorporated in the liposomal membrane exhibited a low degree of emission anisotropy, the values varying between 0.01 and 0.02 . This fact suggests that the fluorophore (Chla macrocycle) is localized in a non-rigid microenvironment. As already mentioned, Chla macrocycle is preferen- 
tially localized at the interface lipid/water, in the vicinity of lipid polar heads and in close contact with water.

The addition of QCT in both types of experiments resulted always in an increase of the emission anisotropy values. This means that the presence of QCT is providing a more rigid microenvironment for Chla, either by inserting in the vicinity of Chla ("quenching sphere") or by influencing the microviscosity of the surrounding phospholipids region (polar heads). The anisotropy increase presents a linear dependence on QCT concentration.

At the same concentration of QCT (in type I experiments), the presence of the anions resulted in lower values for the anisotropy emission. Most evident was in the case of $\mathrm{NO}_{3}{ }^{-}$and $\mathrm{ClO}_{4}{ }^{-}$, chaotropic anions. A possible explanation is the change in the molecular geometry and order of the phospholipids induced by lipid dipole moment alteration due to the interaction with these chaotropic anions. The new molecular geometry influences the QCT localization in the lipid bilayer and interaction with Chla.

In the case of type II experiments (constant QCT concentration), Chla is sensing a more rigid environment when QCT is added to the lipid bilayer in the presence of all anions.

A linear increase of emission anisotropy upon increasing anion concentration was observed in the case of two anions: $\mathrm{SO}_{4}{ }^{-}$and $\mathrm{Cl}^{-}$, stronger increase in the case of $\mathrm{SO}_{4}{ }^{-}$anion. This anion is kosmotrop and therefore the anion could be effective mostly in a region close to water (solvent). Chla, localized at the interface lipid/water in the lipid bilayer, is sensing the microviscosity change related to the presence of increasing concentrations of anions only when QCT is present. These results offer more arguments for the insertion of the QCT at the interface lipid/water in the lipid bilayer.

Both fluorescence and emission anisotropy of Chla in liposomes have been done at physiological $\mathrm{pH}$ and temperature. The results brought evidences for the insertion of QCT at lipid/water interface, in the vicinity of the polar heads of lipids from liposomal bilayer, which is in agreement with previous studies on BLM (Movileanu et al. 2000). The specific influence of the Hofmeister series anions on QCT localization in the lipid bilayer was proved and explained by both methods. The insertion of QCT in the lipid bilayer, at physiological $\mathrm{pH}$ and temperature, has consequences on the microviscosity of lipid moiety in the bilayer. Chla is sensing a more rigid environment when QCT is added to the lipid bilayer and this could be explained by considering a role of QCT similar to cholesterol, as other studies suggested (Tsuchiya et al. 2002; Tarahovsky et al. 2008). Another possible good explanation could be that the addition of QCT to the Chla liposomes is inducing the formation of "lipid rafts" (Tarahovsky et al. 2008) in the close vicinity of Chla. The influence of the Hofmeister series anions on QCT effects is depending on the specific interaction of the anions, either directly on QCT insertion in the bilayer or indirectly, by alteration of molecular geometry of phospholipids.

Chla, used as fluorescence marker for liposomes, was succesfully exploited as sensor at molecular level for revealing specific influence of QCT and anions from Hofmeister series on liposomal membrane.

Acknowledgements. The authors thank Dr. Marcela Elisabeta Bărbîntă-Pătraşcu for the precious help in liposomes preparation. Partial support from CEEX 74/2006 is acknowledged.

\section{References}

Arora A., Byrem T. M., Nair M. G., Strasburg G. M. (2000): Modulation of liposomal membrane fluidity by flavonoids and isoflavonoids. Arch. Biochem. Biophys. 373, 102-109; doi:10.1006/abbi.1999.1525

Barros M. P., Pinto E., Colepicolo P., Pedersén M. (2001): Astaxanthin and peridinin inhibit oxidative damage in $\mathrm{Fe}(2+)$ loaded liposomes: scavenging oxyradicals or changing membrane permeability? Biochem. Biophys. Res. Commun. 288, 225-232; doi:10.1006/bbrc.2001.5765

Bărbîntă-Pătrascu M.E., Țugulea L., Meghea A., Popescu A. (2008) Oxidative stress on liposomes with chlorophyll a monitored by spectral studies. Optoelectronics and Advanced Materials 2, 113-116

Clarke R. J. (1997): Effect of lipid structure on the dipole potential of phosphatidylcholine bilayers, Biochim. Biophys. Acta 278, 1327-269

Clarke R. J., Lüpfert C. (1999): Influence of anions and cations on the dipole potential of phosphatidylcholine vesicles: a basis for the Hofmeister effect. Biophys. J. 76, 2614-2624; doi:10.1016/S0006-3495(99)77414-X

Collins K. D., Washabaugh M. W. (1985): The Hofmeister effect and the behaviour of water at interfaces. Q. Rev. Biophys. 18, 323-422; doi:10.1017/S0033583500005369

Garcia-Celma J., Hatahet L., Kunz W., Fendler K. (2007): Specific anion and cation binding to lipid membranes investigated on a solid supported membrane. Langmuir 23, 10074-10080; doi:10.1021/la701188f

Harwood M., Danielewska-Nikiel B., Borzelleca J. F., Flamm G. W., Williams G. M., Lines T.C. (2007): A critical review of the data related to the safety of quercetin and lack of evidence of in vivo toxicity, including lack of genotoxic/carcinogenic properties. Food Chem. Toxicol. 45, 2179-2205; doi:10.1016/j.fct.2007.05.015

Hertog M. G., Hollman P. C. (1996): Potential health effects of the dietary flavonol quercetin. Eur. J. Clin. Nutr. 50, 63-71

Hollán S. (1996): Membrane fluidity of blood cells. Haematologia (Budap). 27, 109-127

Ilie M., Margină D., Nisiparu L., Ganea C., Katona E. (2009a): Flavonoids effect on the lipid order parameter of peripheral blood mononuclear cells. Rom. J. Biophys. 19, 43-48

Ilie M., Margină D., Katona E., Ganea C., Pencea C., Vladica M., Grădinaru D., Mitrea N., Balalau D. (2009b): Quercetin and Epigallocatechin gallate effect on the lipid order parameter 
of peripheral blood mononuclear cells from diabetes patients. Romanian Biotechnological Letters (in press)

Ilík P., Kouřil R., Kruk J., Myśliwa-Kurdziel B., Popelková H., Strzaka K., Nauš J. (2003): Origin of chlorophyll fluorescence in plants at $55-75^{\circ}$. Photochem. Photobiol. 77, 68-76; doi:10.1562/0031-8655(2003)077<0068: OOCFIP $>2.0$. CO;2

Koynova R., Brankov J., Tenchov B. (1997): Modulation of lipid phase behaviour by kosmotropic and chaotropic solutes, Eur. Biophys. J. 25, 261-274; doi:10.1007/ s002490050038

Lakowicz J.R. (1983): Principles of Fluorescence Spectroscopy, Plenum Press, New York

Metodiewa D., Jaiswal A. K., Cenas N., Dickancaité E., SeguraAguilar J. (1999): Quercetin may act as a cytotoxic prooxidant after its metabolic activation to semiquinone and quinoidal product. Free Radic. Biol. Med. 26, 107-116; doi:10.1016/S0891-5849(98)00167-1

Movileanu L., Neagoe I., Flonta M. L. (2000): Interaction of the antioxidant flavonoid quercetin with planar lipid bilayers. Int. J. Pharm. 205, 135-146; doi:10.1016/S03785173(00)00503-2

New R. R. C. (1990): Liposomes: A Practical Approach (The Practical Approach Series) (Paperback). IRL Press, Oxford University

Oteiza P. I., Erlejeman A. G., Verstraeten S. V., Keen C. L., Fraga C. G. (2005): Flavonoid-membrane interactions: A protective role of flavonoids at the membrane surface? Clin. Dev. Immunol. 12, 19-25; doi:10.1080/ 10446670410001722168

Pawlikowska-Pawlega B., Gruszecki W. I., Misiak L. E., Gawron A. (2003): The study of the quercetin action on human erythrocyte membranes. Biochem. Pharmacol. 66, 605-612; doi:10.1016/S0006-2952(03)00344-7

Pawlikowska-Pawlega B., Gruszecki W. I., Misiak L., Paduch R., Piersiak T., Zarzyka B., Pawelec J., Gawron A. (2007): Modification of membranes by quercetin, a naturally occurring flavonoid, via its incorporation in the polar head group. Biochim. Biophys. Acta 1768, 2195-2204; doi:10.1016/j.bbamem.2007.05.027

Rego A. C., Oliveira C. R. (1995): Dual effect of lipid peroxidation on the membrane order of retinal cells in culture, Arch. Biochem. Biophys. 321, 127-136; doi:10.1006/ abbi.1995.1377

Rietjens I. M., Boersma M. G., van der Woude H., Jeurissen S. M., Schutte M. E., Alink G. M. (2005): Flavonoids and alkenylbenzenes: mechanisms of mutagenic action and carcinogenic risk. Mutat. Res. 574, 124-138; doi:10.1016/ j.mrfmmm.2005.01.028

Ru M. T., Hirokane S. Y., Lo A. S., Dordick J. S., Reimer J. A, Clark D. S. (2000): On the salt-induced activation of lyophilized enzymes in organic solvents: Effect of salt kosmotropicity on enzyme activity, J. Am. Chem. Soc. 122, 1565-1571; doi:10.1021/ja9935198

Saija A., Scalese M., Lanza M., Marzullo D., Bonina F., Castelli F. (1995): Flavonoids as antioxidant agents: importance of their interaction with biomembranes, Free Radic. Biol. Med. 19, 481-486; doi:10.1016/0891-5849(94)00240-K

Sanderson P. W., Lis L. J., Quinn P. J., Williams W. P (1991): The Hofmeister effect in relation to membrane lipid phase stability. Biochim. Biophys. Acta 1067, 43-50; doi:10.1016/00052736(91)90024-3

Scheidt H. A., Pampel A., Nissler L., Gebhardt R., Huster D. (2004): Investigation of the membrane localization and distribution of flavonoids by high-resolution magic angle spinning NMR spectroscopy, Biochim. Biophys. Acta 1663, 97-107; doi:10.1016/j.bbamem.2004.02.004

Strain H. H., Svec W. A. (1966): The Chlorophylls (Eds. L. P. Vernon and G. R. Seely), pp. 21-26, Academic Press, New York, USA

Tarahovsky Y. S., Muzafarov E. N., Kim Y. A. (2008): Rafts making and rafts braking: how plant flavonoids may control membrane heterogeneity. Mol. Cell. Biochem. 314, 65-71; doi:10.1007/s11010-008-9766-9

Tsuchiya H., Nagayama M., Tanaka T., (2002): Membrane-rigidifying effects of anti-cancer dietary factors. Biofactors 16, 45-56; doi:10.1002/biof.5520160301

Ţugulea, L., Pascanu, S. (1996): Chlorophyll a - molecular sensor in model lipid membranes, Romanian J. Biophys. 6, 1-2

Valeur B. (2001): Molecular Fluorescence: Principles and Applications, Wiley-VCH Verlag GmbH, Weinheim

van Dijk C., Driessen A. J., Recourt K. (2000): The uncoupling efficiency and affinity of flavonoids for vesicles. Biochem Pharmacol. 60, 1593-1600; doi:10.1016/S00062952(00)00488-3

Wang L., Tu Y. C., Lian T. W., Hung J. T., Yen J. H., Wu M. J. (2006): Distinctive antioxidant and antiinflammatory effects of flavonols. J. Agric. Food Chem. 54, 9798-9804; doi:10.1021/jf0620719

Zhang Y., Cremer P. (2006): Interactions between macromolecules and ions: the Hofmeister series. Curr. Opin. Chem. Biol. 10, 658-663; doi:10.1016/j.cbpa.2006.09.020

Received: July 7, 2009

Final version accepted: September 24, 2009 